\title{
Max Weber revisited
}

\author{
Die „Wertbeziehung(en)“ der Technikfolgenabschätzung
}

Leonhard Hennen, Institut für Technikfolgenabschätzung und Systemanalyse (ITAS), Karlsruher Institut für Technologie (KIT),

Karlstr.11, 76133 Karlsruhe (leonhard.hennen@kit.edu)

In der aktuellen Debatte um die normativen Grundlagen der Technikfolgenabschätzung (TA) stehen Forderungen nach einer eindeutigen Positionierung der TA in technologiepolitischen Auseinandersetzungen neben Positionen, die an der Rolle der TA als neutraler Beobachter solcher Debatten festhalten wollen. Der Beitrag versucht, durch den Rekurs auf Max Webers kritische Stellungnahmen im ähnlich gelagerten Werturteilsstreit Anfang des 20. Jahrhunderts zur Klärung der Rolle von TA beizutragen. Es wird gezeigt, dass für Max Weber, anders als oft behauptet, Werturteilsfreiheit und engagierte Wissenschaft miteinander vereinbar waren. Für Max Weber war Wissenschaft ohne einen expliziten Bezug auf Werte nicht vorstellbar. Entsprechend kann (und sollte) TA sich ihrer Wertbeziehungen vergewissern, sie auch offensiv vertreten, ohne deshalb den Anspruch werturteilsfreier wissenschaftlicher Analyse aufzugeben.

\section{Max Weber revisited}

The value relation(s) of technology assessment

In the current debate on the normative foundations of technology assessment (TA), demands for TA to explicitly take a political stance in technology policy making coexist with positions holding on to TA's traditional role as a distanced observer of policy making. The present paper tries to clarify TA's role by revisiting Max Weber's critical comments in the so-called "Werturteilsstreit" (Value Judgment Dispute) at the beginning of the $20^{\text {th }}$ century. It is argued that, contrary to what is often stated, for Max Weber scientific analysis refraining from value judgments and engaged scientific intervention are consistent with each other. Scientific analysis, for Weber, is impossible to conceive of without explicit reference to societal values. TA accordingly can (and should) be clear about its own value relations (Wertbeziehungen), and hold on to them in political debate, without necessarily abandoning its ambition of proper and distanced scientific analysis.

Keywords: Werturteilsfreiheit, politics of TA, Max Weber

This is an article distributed under the terms of the Creative Commons Attribution License CCBY 4.0 (https://creativecommons.org/licenses/by/4.0/)

https://doi.org/10.14512/tatup.28.1.27
Die aktuelle Diskussion über die Frage, ob und inwieweit Technikfolgenabschätzung (TA) den Anspruch eines neutralen knowledge broker (Pielke 2007) in umstrittenen technologiepolitischen Fragen aufrechterhalten kann (oder jemals berechtigt war, einen solchen zu behaupten) und nicht vielmehr selbst normativ begründete Positionen beziehen sollte (bzw. implizit immer schon bezogen hat), ist angesichts der vielfältigen normativen Bezüge, in denen TA als hybride Praxis zwischen Wissenschaft und Politik steht, mehr als berechtigt. Feststellbar ist dabei eine unvermittelte Koexistenz von starken Plädoyers für offen normative technologiepolitische Stellungnahmen einerseits und einem (irgendwie gearteten) Festhalten an der Rolle des wissenschaftlich distanzierten Beobachters andererseits. ${ }^{1}$ In dieser Situation kann die Erinnerung an eine ähnlich gelagerte historische Diskussion hilfreich sein: den sogenannten „Werturteilsstreit“ Anfang des 20. Jahrhunderts. Bekanntlich war der Ausgangspunkt dieses Streites - durchaus ähnlich der aktuellen Diskussion in der TA-Community - die Frage nach der (Un-)Möglichkeit, konkrete werthaltige Handlungsempfehlungen an die Politik aus der wissenschaftlichen Analyse abzuleiten. Max Weber stellte sich in dieser Diskussion gegen die sogenannten Kathedersozialisten, nach deren Auffassung es Aufgabe der Wissenschaft (d.h. im vorliegenden Fall der Nationalökonomie) sei, in der um die Jahrhundertwende drängenden sozialen Frage nicht nur Stellungnahmen zur Sozialpolitik zu erarbeiten. Sie waren darüber hinaus der Ansicht, dass sich werthaltige, praktisch-politische Programme wissenschaftlich begründen ließen. Ihre Zielsetzung war es dabei, durch eine verbesserte Lage der Arbeiterklasse gerade dem Erstarken sozialistischer Kräfte in der Politik entgegenzuwirken. Die in diesem Kontext entstandenen Arbeiten und Stellungnahmen Max Webers haben für die wissenschaftstheoretische Grundlegung der Sozialwissenschaften eine wichtige Rolle gespielt. Sein auch in der TA diskutiertes Konzept der Werturteilsfreiheit (oft fälschlich verkürzt zu Wertfreiheit) der Wissenschaft steht hierfür. Max Weber hat aber daneben mit dem weniger rezipierten Begriff der Wertbeziehung als wesentlicher Voraussetzung (sozial-)wissenschaftlicher Praxis eine

1 Siehe dazu die Beiträge auf der 2. Europäischen TA-Konferenz in Cork im Mai 2017 (Hennen und Nierling 2018), der österreichischen TA18 im Juni 2018, und der letzten NTA-Konferenz in Karlsruhe im November 2018 sowie die Beiträge in diesem TATuP-Thema. 
durchaus komplexe Analyse der normativen Bedingungen von Wissenschaft vorgelegt. Ziel des vorliegenden Beitrages ist es, mittels der Lektüre ${ }^{2}$ dieser Analyse durch die TA-Brille die aktuelle Debatte nicht unbedingt durch neue Argumente anzureichern, aber die vorliegenden Argumente mittels einer Art Verfremdungs-Effekt in einem anderen, vielleicht aufschlussreichen Licht zu betrachten.

\section{Der praktische Wert „werturteilsfreier“ Wissenschaft}

Webers Grundposition ist bekanntlich gekennzeichnet durch eine strikte Trennung politischer (moralisch-praktischer) und wissenschaftlicher (analytischer) Tätigkeit, wie er sie etwa in seinem berühmten Aufsatz „Wissenschaft als Beruf“ (Weber 1919) begründet. Wissenschaft weist sich hier insofern als ,werturteilsfrei“ aus, als es keinen Weg gibt, aus dem „Sein“ bzw. aus der Analyse empirischer sozialer Verhältnisse, ein „Sollen“, etwa eben die Wünschbarkeit bestimmter sozialpolitischer Maßnahmen, zu begründen. Soweit, so bekannt, und es läge nun der Schluss nahe, der ja später auch oft gezogen worden ist, dass deshalb wissenschaftliche Politikberatung ihre Legitimität allein aus der Beschränkung auf die Rolle des neutralen, wertfreien knowledge broker gewinnen kann. Dass Webers Position, anders als oft behauptet, aber nicht (jedenfalls nicht umstandslos) als Referenz für Neutralität (in der TA) taugt, er die Sache mit den Werten jedenfalls etwas komplizierter sah, macht den Versuch eines Max Weber revisited für die aktuelle Debatte interessant. Die vor dem analytischen Zugriff auf die Wirklichkeit liegenden normativen Voraussetzungen wissenschaftlicher Arbeit nennt Weber ihre der (quasi technischen) Aufklärung über die zur Erreichung eines gegebenen „Zweckes“ (über dessen normative Begründung wissenschaftlich allerdings nichts auszusagen ist) geeigneten „Mittel“ und die dabei in Kauf zu nehmenden „Nebenfolgen“ (Weber 1988, S. 607). Das nennt Max Weber „Klarheit“ als den praktischen (politischen) Wert wissenschaftlicher Erkenntnis (ebd.) und dürfte in den Ohren des TA-Praktikers durchaus geläufig klingen. Der praktische Wert der Wissenschaft geht aber über das rein technische Zweck-Mittel-Verhältnis hinaus. Zwar kann wissenschaftlich nicht über die, die Praxis leitenden Werte (Zwecke) entschieden werden, aber die wissenschaftliche Wertediskussion kann dem Praktiker dabei helfen ,,sich selbst Rechenschaft zu geben über den letzten Sinn seines eigenen Tuns“ (ebd., 608). D. h. es kann Klarheit über die inneren Konsequenzen der Entscheidung zu einer bestimmten praktischen Stellungnahme geschaffen werden: Auf welchen Wertannahmen beruht sie (und welche schließt sie damit aus)? Was bedeutet es, sich praktisch durch diese anleiten zu lassen? Welche anderen Werte werden dadurch notwendig verletzt oder ignoriert und mit welchen Folgen? „Ihr dient, bildlich geredet, diesem Gott und kränkt jenen anderen, wenn ihr euch für diese Stellungnahme entschließt. Denn ihr kommt notwendig zu diesen und diesen letzten inneren, sinnhaften Konsequenzen, wenn ihr euch treu bleibt.“ (Weber 1988, S. 608) Dieser Beitrag der Wissenschaft zum praktischen Leben scheint Weber ,nicht so sehr wenig zu sein“(ebd.). Und das wäre auch in Bezug auf TA festzuhalten: Unter Enthaltsamkeit bzgl. eigener politischer Stellungnahmen den Protagonisten (technologie-)politischer Debatten zu Klarheit gegenüber ihrer eigenen Stellungnahme zu verhelfen, möglicherweise mit der Konsequenz diese zu relativieren, um auch den anderen Wertgesichtspunkten (Göttern) zu ihrem Recht zu verhelfen, scheint

\section{Im Sinne Webers ist Wissenschaft kein Selbstzweck, sondern legitimiert sich gesellschaftich durch ihren praktischen Wert.}

\begin{abstract}
„Wertbeziehung“. Es ist dieser Begriff mit dem Weber die nicht nur unvermeidliche, sondern im Hinblick auf die Gewährleistung praktischer Relevanz oder gar des Sinns wissenschaftlicher Tätigkeit notwendige normative Prägung von Wissenschaft anspricht - und zwar durchaus im Sinne engagierter Wissenschaft.

Zunächst einmal ist festzuhalten, dass im Sinne von Webers Ausführungen in „Wissenschaft als Beruf“ Wissenschaft natürlich kein Selbstzweck ist, sondern sich gesellschaftlich durch ihren praktischen Wert legitimiert (sozusagen ihr grundlegender Wertbezug), der darin besteht, dass sie einen Beitrag zur Lösung gesellschaftlicher Probleme leistet. Dieser wiederum besteht in
\end{abstract}

2 Bezug genommen wird auf drei zentrale Texte Webers: „Die ,Objektivität sozialwissenschaftlicher und sozialpolitischer Erkenntnis" (1904), „Der Sinn der ,Wertfreiheit' der soziologischen und ökonomischen Wissenschaften“ (1917) und "Wissenschaft als Beruf" (1919). nicht eben wenig zu sein, wenn auch die Aussichten, dass dies gelingt, je nach politischer Wetterlage, nicht immer gut sein mögen. Und dies impliziert - wie ich meine - jedenfalls mehr als distanziertes Knowledge-Brokering, sondern ist engagierte Gesellschaftsberatung im Sinne sich einmischender Aufklärung der Praxis. Max Weber hatte eine Begründung der praktischen Relevanz der Sozialwissenschaften (bzw. in seiner damaligen Diktion der Kulturwissenschaften) im Sinne. Aus heutiger TA-Sicht würde man ergänzen, dass zu solcher „Klarheit“ transdisziplinäre Forschung, d. h. das Zusammenwirken einer Vielzahl wissenschaftlicher Disziplinen im Dialog mit einem weiten Spektrum gesellschaftlicher Interessen und Erwartungen, notwendig ist. Ohne diesen Aufwand ist „Klarheit“ im Sinne der öffentlichen Selbstvergewisserung über die Konsequenzen der verschiedenen möglichen Stellungnahmen zu einem praktischen Problem, nicht zu erlangen. 


\section{Die wertbezogene Konstitution des Gegenstands wissenschaftlicher Analyse}

Werturteilsfreiheit von Wissenschaft impliziert, wie oben gezeigt, zunächst also durchaus (und nicht nur nebenbei, sondern als gesellschaftliche Aufgabe der Wissenschaft) die Möglichkeit einer wissenschaftlichen Diskussion der Beziehung von Mitteln und Zwecken sowie der, die letzteren begründenden Wertideen in praktisch-aufklärender Absicht. Wertfrei ist sie daher nicht. Sie kann es darüber hinaus aber auch in einem, ihre epistemologischen Voraussetzungen betreffenden Sinn nicht sein. Neben den „Regeln der Logik und Methodik“ zählt zu den Voraussetzungen wissenschaftlicher Arbeit, über die der Wissenschaftler sich Rechenschaft ablegen muss, ,[...] dass das, was bei der Wissenschaft herauskommt, wichtig im Sinne von, wissenswert" sei“ (Weber 1988, S. 599). Und hier, so Weber, ,[...] stecken nun offenbar alle unsere Probleme darin" (ebd.), weil diese Voraussetzung nicht wiederum wissenschaftlich beweisbar ist. „Keine Wissenschaft ist absolut voraussetzungslos, und keine kann für den, der diese Voraussetzungen ablehnt, ihren eigenen Wert begründen“" (ebd., S. 610). In Bezug auf TA wäre also zunächst festzuhalten, dass natürlich auch sie ihre eigenen Grundlagen oder ihre Mission nicht aus sich selbst begründen kann. Vielmehr wird hier eine vorwissenschaftliche (politische) Entscheidung getroffen, und zwar nicht nur dann, wenn man sich für eine dezidiert politisch agierende und Stellung beziehende TA entscheidet (Delvenne und Parotte 2019), sondern auch dann, wenn man sich selbst als neutralen Beobachter verstehen möchte. Die Entscheidung für Neutralität in praktisch-politischen Debatten wäre so gesehen - unabhängig von der Frage, ob Neutralität möglich ist - als eine Entscheidung über die (politische) Rolle oder das Selbstverständnis von TA zu begreifen, die aus praktisch politischen Überlegungen getroffen wird und etwa auch als „foundational myth“ der TA taktisch motiviert sein kann (Torgersen 2019).

Mit den vorwissenschaftlichen Voraussetzungen verbindet sich eine wertende Beziehung von Wissenschaft auf Realität, und diese bestimmt auch den Objektbezug jeder wissenschaftlichen Untersuchung. „Wertbeziehung“ im engeren Sinne impliziert ,[...] die philosophische Deutung desjenigen spezifisch wissenschaftlichen Interesses, welches die Auslese und Formung eines wissenschaftlichen Objektes beherrscht" (Weber 1988, S. 511). Es ist also eine außerwissenschaftliche Reflexion, die das Erkenntnisinteresse des Wissenschaftlers begründet, und diese betrifft nicht nur die „Auslese“ des Gegenstandes als des zu untersuchenden Problems, sondern auch dessen „Formung“, d. h. wie dieser Gegenstand grundsätzlich aufgefasst wird bzw. unter welchem Gesichtspunkt er untersucht wird. Weber spricht in dieser Hinsicht auch von der „Kulturbedeutung“ des Gegenstandes und der Untersuchung. Durch die Wertbeziehung wird die wissenschaftliche Untersuchung sozusagen in praktische Beziehung zur Kulturwirklichkeit gesetzt, die durch Werte konstituiert wird. Die Komplexität und Vielfalt der kulturellen Wirklichkeit macht eine vorwissenschaftlich wertgeleitete Auswahl der Fragestellung und der Perspektive nötig, weil schon eine einfache Beschreibung der Elemente der Wirklichkeit und erst recht die Aufdeckung von ursächlichen Zusammenhängen ohne eine solche Entscheidung unmöglich ist. Es kommt darauf an, die Wirklichkeit unter einem Aspekt zu erfassen, der „kulturbedeutend" ist, also möglichst etwas Wesentliches oder Typisches der soziohistorischen Realität erfasst (ebd., S. 180, 182). Auch dieser Gedanke lässt sich aus dem Kontext wissenschaftstheoretischer Begründung der „Kulturwissenschaften“ in die Welt der TA übertragen: Problemorientierte Forschung gewinnt die Aspekte, unter denen Wirklichkeit thematisch wird, aus den Problemen, die die gesellschaftliche Diskussion und die Politik bestimmen.

\section{In Bezug auf TA ist festzuhalten, dass sie ihre eigenen Grund- lagen oder ihre Mission nicht aus sich selbst begründen kann.}

Webers Formulierungen legen nahe, dass es darauf ankommt, diejenige Problemperspektive einzunehmen, die sozusagen gesamtgesellschaftlich relevant ist, d. h. den Kern gesellschaftlicher Problemwahrnehmung trifft. Dabei würde sich natürlich unmittelbar die Frage stellen, wie dies zu bestimmen wäre. Klar ist zunächst, dass eine solche Bestimmung selbst wieder dem kulturellen Wandel unterliegt. Mit dem kulturellen Wandel ändern sich auch die Forschungsgegenstände, weil die Gesellschaft sich sozusagen selbst unter anderen Problemgesichtspunkten thematisiert: ,[I]rgendwann wechselt die Farbe: die Bedeutung der unreflektiert (Hervorhebung des Autors) verwerteten Gesichtspunkte wird unsicher, der Weg verliert sich in der Dämmerung“ (ebd., S. 214). Damit wechselt nun auch die Wissenschaft ihren „Begriffsapparat“ und sie ,zieht jenen Gestirnen nach, welche allein ihrer Arbeit Sinn und Richtung zu weisen vermögen“ (ebd.). Wissenschaft unterliegt also selbst dem soziokulturellen Wandel, den sie untersuchen will, und ist geprägt (mehr noch: muss sich, um sinnvoll zu sein, prägen lassen) von Wertideen, die im Idealfall aus den wesentlichen, drängenden Fragen der jeweiligen Gegenwart gespeist sind. Damit ist eine normative Imprägnierung gerade einer Forschung in politikberatender Absicht immer schon durch den Zeitgeist gegeben, wie sich etwa an den für TA zentralen Konzepten wie Risiko oder Nachhaltigkeit festmachen ließe (Grunwald 2018). Eine solche Imprägnierung scheint wohl in der Tat aus Webers Sicht nicht nur unvermeidbar, sondern auch wünschbar. Dies sollte aber sozusagen nicht auf Kosten der Schärfe des kritischen Blickes geschehen. Wertbezug heißt im Sinne Webers nicht Übernahme eines vermeintlichen gesellschaftlichen Konsenses darüber, was das drängende Problem der Zeit oder im Interesse des Gemein- 
wohls sei oder - wie etwa bei den von Weber kritisierten Kathedersozialisten - die Orientierung am ,Daseinsinteresse des Staates" als letztem Wert wissenschaftlicher Erkenntnis (Weber 1988, S. 539). Im Gegenteil, man dürfe sich nicht „,bei irgendeiner [...] durch Konvention geschaffenen Selbstverständlichkeit gewisser noch so weit verbreiteter praktischer Stellungnahmen wissenschaftlich beruhigen“ (ebd., S. 502). Für Weber ist es „die spezifische Funktion der Wissenschaft", dass sie sich kritisch zur Wirklichkeit verhält, ,indem ihr das konventionell Selbstverständliche zum Problem wird“" (ebd.).

Das heißt, auch Technikfolgenforschung entscheidet über die sie leitenden Wertgesichtspunkte nicht unabhängig von technologiepolitischen Debatten und den hier herrschenden Positionen, sie muss diese im Gegenteil in Rechnung stellen. Aber sie muss dann die sie leitenden Wertideen selbst setzen und vertreten. Damit ist sowohl die Bemühung um eine Forschungsperspektive, die von einem breitest möglichen politischen Konsens gedeckt ist (wie z. B. in der politikberatenden Praxis des Büros für Technikfolgen-Abschätzung beim Deutschen Bundestag (TAB) durch die Rolle der parlamentarischen Berichterstatter intendiert), wie auch die Wahl einer spezifischen Auftraggeberoder Stakeholder-Perspektive, schließlich aber auch die Begründung einer eigenen kritischen Forschungsperspektive legitimiert, solange diese Wahl als vorwissenschaftliche transparent bleibt und die Untersuchung selbst gemäß den Standards werturteilsfreier Wissenschaft durchgeführt wird.

\section{Wissenschaft und politisches Engagement}

Ein in der aktuellen TA-Debatte wichtiges Argument gegen das Postulat der wie auch immer verstandenen politischen Neutralität der TA bzw. besser der Distanziertheit gegenüber der politischen Kontroverse ist es, dass Wertfreiheit der praktischen wissenschaftlichen Arbeit nicht möglich sei, schon weil jeder TA-Praktizierende ja mit einer gewissen nicht einfach ausschaltbaren persönlichen Einstellung zur vorliegenden Fragestellung an die Arbeit herangehe (siehe hierzu z. B. das von Bauer und Kastenhofer 2019 herausgearbeitete Repertoire von „Rollen“ in der TA-Praxis). Interessanterweise zeigt sich, dass auch Weber als Verfechter der Werturteilsfreiheit nicht nur nicht blauäugig gegenüber solchen Beobachtungen war, sondern ein praktisches Verhältnis oder Wollen in der Sache geradezu als Impetus der wissenschaftlichen Arbeit ansah. Anlässlich der Übernahme der Redaktion der Zeitschrift Archiv für Sozialwissenschaften und Sozialpolitik betont Weber, dass aufgrund des notwendigen Bezugs der Sozialwissenschaften auf praktische Fragen, schon „die bloße Anerkennung eines wissenschaftlichen Problems in Personalunion steht mit einem bestimmt gerichteten Wollen lebendiger Menschen." (Weber 1988, S. 158) Ein solches Wollen, eine bestimmte „Wertidee“ (ebd., S. 159), welche die Problemwahrnehmung und auch die Wahrnehmung der Dringlichkeit ihrer Lösungen leitet, hält er für unerlässlich, wenn es um eine kritische Auseinandersetzung mit Fragen praktischer Politik geht. Die Kritik praktischer Politik, worin er - hier nun sozusagen die Grenzen der reinen Forschung, um die es ihm in seinen methodologischen Arbeiten geht, überschreitend - durchaus die Aufgabe der Zeitschrift sah, geht immer von einem die eigenen Ideale leitenden „Wertaxiom“ aus und wendet sich gegen andere (ebd., S. 156 f.).

In diesem Zusammenhang beschreibt Weber den kathedersozialistischen Geist der Gruppe von Wissenschaftlern um das Archiv als legitimen praktischen Antrieb und auch Grund der anhaltenden Bedeutung der Zeitschrift. Dieses gemeinsame Ethos bestand im Interesse an der Hebung des Lebensstandards der Arbeiter auf der Grundlage kapitalistischer Wirtschaftsform. Aus dieser Perspektive wurde die Politik kritisch kommentiert und wurden praktisch-politische Vorschläge erarbeitet. ${ }^{3}$ Es kommt aber bei der wissenschaftlich unterfütterten politischen Kritik darauf an, ,in jedem Augenblick den Lesern und sich selbst scharf zum Bewusstsein zu bringen, welches die Maßstäbe sind, an denen die Wirklichkeit gemessen und aus denen das Werturteil abgeleitet wird“" (ebd., S. 157). Eine wissenschaftlich fundierte, praktische Kritik von Politik ist ohne einen eigenen außerwissenschaftlichen Standpunkt nicht möglich. Weber geht an dieser Stelle ganz offensichtlich über die Aufgabe der reinen Wertediskussion (s. o.) hinaus, die sich ja noch distanziert zu unterschiedlich begründeten Wertgesichtspunkten hält, und vertritt als Aufgabe einer wissenschaftlichen Zeitschrift für politische Fragen ein durchaus selbst wertgeleitetes Eingreifen in politische Debatten. Man könnte demnach vielleicht von zwei unterschiedlichen Modi ,politikberatender“ Wissenschaft sprechen, einem distanziert aufklärenden und einem engagiert eingreifenden. Es kommt bei letzterem aber darauf an, dass der eigene (praktisch-politisch, ethisch, weltanschaulich begründete) Wertgesichtspunkt klar erkennbar bleibt.

In Webers Konzept der Wertbeziehung hat ein über das reine Forschungsinteresse hinausgehender Wertbezug also durchaus einen Platz. Dieser kommt dann zum Tragen, wenn sich Wissenschaft (wie im vorliegenden Falle einer Zeitschrift für Sozialpolitik und nicht allein für Sozialwissenschaft) praktisch-politisch äußert. Die Autoren des Archivs für Sozialpolitik und Sozialwissenschaft teilen das Interesse an sozialpolitischen Problemen (die Lage der Arbeiterklasse) und prägen durch die „Wahlverwandtschaft ähnlicher Ideale“ der Zeitschrift einen bestimmten „Charakter“ in der Behandlung politischer Fragen auf (ebd., S. 158 f.). Einen solchen „Charakter“ sieht Weber offensichtlich als legitime Motivation politisch engagierter Wissenschaft an, unterscheidet diesen aber scharf von einer „Tendenz“, die sozusagen der Befassung mit solchen Problemen von vornherein eine politische Färbung aufprägt und etwa die Zeitschrift auf ein politisches Lager festlegen würde.

3 Weber stellt sich also nicht eigentlich gegen die sogenannten Kathedersozialisten, sondern sieht hier einen besonderen Wertbezug realisiert. Er stellt sich gegen die Verwechslung eines „wertvollen“ Bezugs auf die Realität als Forschungsgegenstand und die unzulässige stillschweigende Unterstellung, dass sich diese Werte aus der wissenschaftlichen Analyse begründen ließen. 
Es liegt auf der Hand, analog für die TA-Community Elemente eines solchen „Charakters“ zu konstatieren: Ein gewisses kritisches Verhältnis zur Frage der zivilisatorischen Bedeutung wissenschaftlich-technischer Innovation gehört sicherlich ebenso zum (legitimen) Selbstverständnis der Zunft wie etwa die Präferenz für inklusorische/partizipative Verfahren der Problemidentifikation und -bearbeitung. Der TA(-Community) werden Wissenschaft und Technologie immer unter bestimmten Wertgesichtspunkten zum Problem: Diese können solche sein, die durch einen weitgehenden gesellschaftlichen Konsens über die Kulturbedeutung einer Frage, wie etwa der des Klimawandels, getragen sind; aber auch solche, die dezidiert als gegen den Mainstream gewandte Fragen etwa nach ökologischer Nachhaltigkeit oder sozialer Gerechtigkeit gewählt sind. Auch folgt TA in der Bearbeitung solcher Probleme bestimmten, selbst nicht wissenschaftlich begründbaren (und etwa populistischen Wertideen diametral entgegenstehenden) Idealen, nämlich jenen wissenschaftlich-rationaler Analyse und demokratischer Deliberation. Die politische Aufgabe und Rolle von TA (zentraler Aspekt der „Politics of TA“, s. Hennen und Nierling 2019) ist es durchaus, diese Ideale zu vertreten und in der wissenschaftlich-beratenden Arbeit durchzusetzen. Dies impliziert aber keine apriorische Stellung zu praktisch-politischen Lösungsvorschlägen und in jedem Fall muss dort, wo die Entscheidung für eine Wertidee die Untersuchung motiviert, etwa dann, wenn ein bestimmtes Konzept von Nachhaltigkeit als normative Grundentscheidung der Neutralität darin liefern auch für die Haltung des ,providing something to everybody" Anschauungsmaterial, so etwa im Falle des frühen US-Amerikanischen Office of Technology Assessment (OTA) (Torgersen 2019, S. 59).

\section{TA als „Kulturwissenschaft"?}

Zum Schluss soll eine Frage nicht vermieden werden, mit der die Übung einer Fruchtbarmachung der ca. 100 Jahre alten wissenschaftstheoretischen Überlegungen Webers für die TA steht und fällt - und die hier nicht ausreichend thematisiert werden kann: Natürlich ist TA nicht mit Kulturwissenschaften im Sinne Webers gleichzusetzen. Es geht hier nicht um geistes- und sozialwissenschaftliche Forschung, sondern um inter- oder transdisziplinäre Forschung, orientiert an gesellschaftlich definierten Problemlagen. Dennoch kann die kulturwissenschaftliche Perspektive Webers - wie hoffentlich klargeworden ist - für unsere Praxis erhellend sein. Für Weber ist alle kulturwissenschaftliche Forschung problemorientierte Forschung, indem sie nur insofern Bedeutung gewinnt, als sie Kulturprobleme zum Gegenstand ihrer Analyse wählt, sich also bei der Auswahl der Fragestellung und des Gegenstandes davon leiten lässt, was kulturell bedeutsam ist. Hierin ist natürlich immer eine Wertung enthalten: eine Orientierung an Kulturwerten. Festgehalten werden kann jedenfalls, dass sich problemorientierter Forschung vom

\section{Eine wissenschaftlich fundierte, praktische Kritik}

\section{von Politik ist ohne einen eigenen außerwissenschaftlichen Standpunkt nicht möglich.}

die Analyse leitet, die zugrundeliegende Wertidee immer klar herausgearbeitet sein. Und was die scheinbar beste Lösung für sagen wir - eine nachhaltige Energiewirtschaft im Einzelnen ist, muss in jedem Fall unter Berücksichtigung verschiedener Werte (Wertediskussion, s. o.) Gegenstand der TA-Untersuchung sein und nicht schon als Tendenz vorgegeben.

Ebenso fatal wie eine wissenschaftliche Verschleierung der eigenen wertgebunden Position wäre es nach Weber aber ,wie es nur allzu oft geschieht, durch unpräzises Ineinanderschieben von Werten verschiedenster Art sich um die Konflikte zwischen den Idealen herumzutäuschen und ,jedem etwas bieten ' zu wollen.“ (Weber 1988, S. 157) Die Handlungsmaxime ,jedem etwas bieten zu wollen“, in welchem Sinne Neutralität als Kennzeichen politikberatender TA ja oft vertreten oder kritisiert wird, wäre damit sozusagen als die schlechte Variante von Distanziertheit zu verstehen, eine Form von Beobachterposition, die gerade nicht aufklärt, sondern die Komplexität und Konflikthaftigkeit der für ein vorliegendes Problem relevanten Wertgesichtspunkte verschleiert. Die Geschichte der TA und die Rolle des Konzepts
Typus TA, ebenso wie den Kulturwissenschaften, die Aufgabe stellt, die Wirklichkeit unter den Problemgesichtspunkten zu erfassen, die von kultureller Bedeutung sind, also unsere gegenwärtige Problemwahrnehmung prägen oder - und hier käme der Pluralismus von Werten zum Tragen - nach verschiedenen konkurrierenden Auffassungen prägen sollten. TA ist insofern Kulturwissenschaft im Sinne Webers, als uns Technik ja nicht in erster Linie unter dem technischen Aspekt ihres Funktionierens problematisch wird (das ist Sache des Ingenieurs), sondern hinsichtlich ihrer Kulturbedeutung, die im weiten Sinne verstanden in der gesellschaftlichen Auseinandersetzung über Fragen der Umweltauswirkungen (Umwelt als Kulturgut) ebenso zur Debatte steht wie in der Frage der potenziellen Veränderung sozialer Interaktionsformen (etwa durch Social Media).

\section{Literatur \\ Bauer, Anja; Kastenhofer, Karen (2019): Policy advice in technology assessment. Shifting roles, principles and boundaries. In: Technological Forecasting and Social Change 139, S. 32-41.}


Delvenne, Pierre; Parotte, Celine (2019): Breaking the myth of neutrality. Technology assessment has politics, technology assessment as politics. In: Technological Forecasting and Social Change 139, S. 64-72.

Grunwald, Armin (2018): Versteckte Normativitäten in der Technikfolgenabschätzung? Vortrag bei der Konferenz TA18 „Technikfolgenabschätzung und Normativität. An welchen Werten orientiert sich TA?", Wien, 11. Juni 2018. Hennen, Leonhard; Nierling, Linda (2019): The politics of TA. Introduction to the special issue of technological forecasting and social change. In: Technological Forecasting and Social Change 139, S. 17-22.

Pielke Jr., Roger (2007): The honest broker. Making sense of science in policy and politics. Cambridge: Cambridge University Press.

Torgersen, Helge (2019): Three myths of neutrality in TA. How different forms of TA imply different understandings of neutrality, In: Technological Forecasting and Social Change 139, S. 57-63.

Weber, Max (1904): Die „Objektivität“ sozialwissenschaftlicher und sozialpolitischer Erkenntnis. In: Ders. (1988): Gesammelte Aufsätze zur Wissenschaftslehre. Tübingen: J. C. B. Mohr, S. 146-214.

Weber, Max (1917): Der Sinn der „Wertfreiheit“ der soziologischen und ökonomischen Wissenschaften. In: Ders. (1988): Gesammelte Aufsätze zur Wissenschaftslehre. Tübingen: J. C. B. Mohr, S. 489-540.

Weber, Max (1919): Wissenschaft als Beruf. In: Ders. (1988): Gesammelte Aufsätze zur Wissenschaftslehre. Tübingen: J. C. B. Mohr. S. 582-613.

Weber, Max (1988): Gesammelte Aufsätze zur Wissenschaftslehre. Tübingen: J.C.B. Mohr.

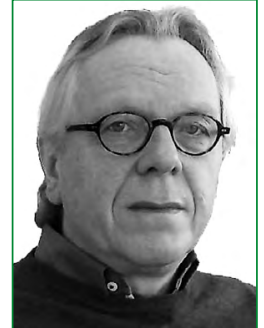

\section{DR. LEONHARD HENNEN}

arbeitet als Senior Scientist am ITAS und ist als Soziologe in zahlreichen internationalen Projekten zu Konzepten und Methoden der TA involviert. Seit 2005 ist er Koordinator der European Technology Assessment Group (TA Studien im Auftrag des Europäischen Parlamentes), von 1991 bis 2005 war er Projektmanager im Büro für TechnikfolgenAbschätzung beim Deutschen Bundestag.

\section{Zukünftige TATuP-Themen auf einen Blick ...}

Erscheinungstermine, Inhalte, Thema-Herausgeberinnen und -Herausgeber sowie die Veröffentlichungstermine der Call for Abstracts in kommenden TATuP-Ausgaben:

\section{TATuP 2019, Heft 2 (Juli)}

Thema: Digitalisierung und Entwicklung im Globalen Süden. Fortschritt durch Technologie?

Thema-Herausgeberinnen: Jessica Heesen, Laura Schelenz, Kerstin Schopp und Maria Pawelec (alle IZEW, Universität Tübingen)

Call for Abstracts: geschlossen

\section{TATuP 2019, Heft 3 (Dezember)}

Thema: Energiezukünfte: wissen, beraten, gestalten

Thema-Herausgeberin und -Herausgeber: Lisa Nabitz, Witold Roger Poganietz und Dirk Scheer (alle ITAS, KIT)

Call for Abstracts: geschlossen

\section{TATuP 2020, Heft 1 (April)}

Thema: Cybersicherheit

Thema-Herausgeber: Karsten Weber (OTH Regensburg),

Markus Christen (Universität Zürich) und Dominik

Herrmann (Universität Bamberg)

Call for Abstracts: ca. Mitte Mai 2019

\section{TATuP 2020, Heft 2 (Juli)}

Thema: Integrated Infrastructures

Thema-Herausgeber: Christian Büscher (ITAS, KIT), Bert Droste-Franke (EA European Academy) und Michael Ornetzeder (ITA, Wien)

Call for Abstracts: ca. Mitte September 2019

\section{TATuP 2020, Heft 3 (Dezember)}

Thema: Demokratie und Technikfolgenabschätzung

Thema-Herausgeber: Armin Grunwald (ITAS, KIT) und Thomas Saretzki (Leuphana Universität Lüneburg)

Call for Abstracts: ca. Februar 2020

\section{TATuP 2021, Heft 1 (April)}

Thema: Urbane Zukünfte/Smart Cities

Thema-Herausgeberin und -Herausgeber: Cordula Kropp (Universität Stuttgart) und Ulrich Ufer (ITAS, KIT)

Call for Abstracts: ca. Mai 2020 